\title{
Cerebral Small-Vessel Diseases: A Look Back from 1991 to Today
}

\author{
Lina Grosset ${ }^{\mathrm{a}, \mathrm{b}} \quad$ Eric Jouvent ${ }^{\mathrm{a}} \mathrm{b}, \mathrm{c}$

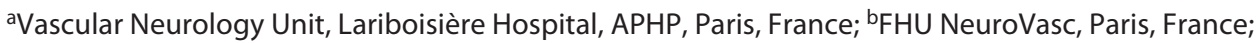 \\ 'Université de Paris, Paris, France
}

\section{Keywords}

Cerebral small-vessel disease - Lacunes - Lacunar stroke . Lacunar syndrome $\cdot$ White matter hyperintensities $\cdot$ Brain $M R I \cdot$ Diffusion MRI

\begin{abstract}
Background: Cerebral small-vessel diseases (cSVDs) encompass a number of causes involving, but not limited to, alterations in the intracranial microvasculature, leading to the accumulation of brain tissue damage and the development of various degrees of cognitive impairment, behavioral alterations, gait instability, and localization signs, often associated with the occurrence of ischemic or hemorrhagic strokes. Summary: In 2021, although key questions remain unanswered, there is general agreement on the construct, its main pathophysiological bases, and the terms used to describe its main clinical and radiological features. However, this has not always been the case, and the 30th anniversary of Cerebrovascular Diseases is an opportunity to look back from 1991 to the present to understand how a number of features, sometimes considered independent, have been progressively brought together by successive scientific breakthroughs, gradually leading to the definition of the now widely accepted concept of cSVDs. Key Messages: In the course of this journey, we will detail with particular attention the role of what we consider 2 crucial events: the advent of cerebral MRI and the building of large cohorts with monogenic forms of small-vessel disease of the brain.
\end{abstract}

(c) 2022 S. Karger AG, Basel

\section{Introduction}

The concept of cerebral small-vessel disease (cSVD) is now widely recognized. The publication in 2013 of the STRIVE criteria [1], the result of an international effort to homogenize cSVD terminology for clinical and research questions, was critical in achieving professional agreement on the fundamentals of cSVD and the dissemination of commonly accepted terms to describe their clinical and radiological aspects. A number of excellent reviews have been published recently [2], and our goal is not to write another one but rather to take advantage of the 30th anniversary of Cerebrovascular Diseases to put the current concepts in perspective with knowledge in the field dating back to 1991. Given the breadth of the subject, we have chosen to focus on ischemic forms of cSVD and will voluntarily leave aside the hemorrhagic markers of arteriolosclerosis and cerebral amyloid angiopathy.

In this review, we wish to show how, in the last 30 years, two major events occurred that led to a profound revision of the premises of what we know today as cSVD: (1) the advent of MRI with a particular emphasis on diffusion-weighted sequences, which gave a much clearer picture of the radiological spectrum of cSVD and (2) the identification of mutations of the NOTCH3 gene as the cause of cerebral autosomal dominant arteriopathy with

This article was published in celebration of the 30th anniversary of the inception of Cerebrovascular Diseases (1991-2021). 
Fig. 1. Brain MRI compared to the CT scan for evaluating cSVD in daily clinical practice. Comparison of registered axial slices of the CT scan and MRI in a patient admitted in the stroke unit for cSVD-related ischemic stroke. Please note that the quality of the CT scan is far better than it was in 1991. The FLAIR MRI sequences shows periventricular WMH which are barely visible on the CT scan. Center: the DWI sequence shows a recent small subcortical infarct in the right lenticular nucleus, barely visible on the CT scan. Right: susceptibility-weighted imaging sequence showing a microbleed in the brainstem, not visible by definition on the CT scan. cSVD, smallvessel disease; FLAIR, fluid-attenuated inversion recovery; DWI, diffusion-weighted imaging; $\mathrm{WMH}$, white matter hyperintensities.

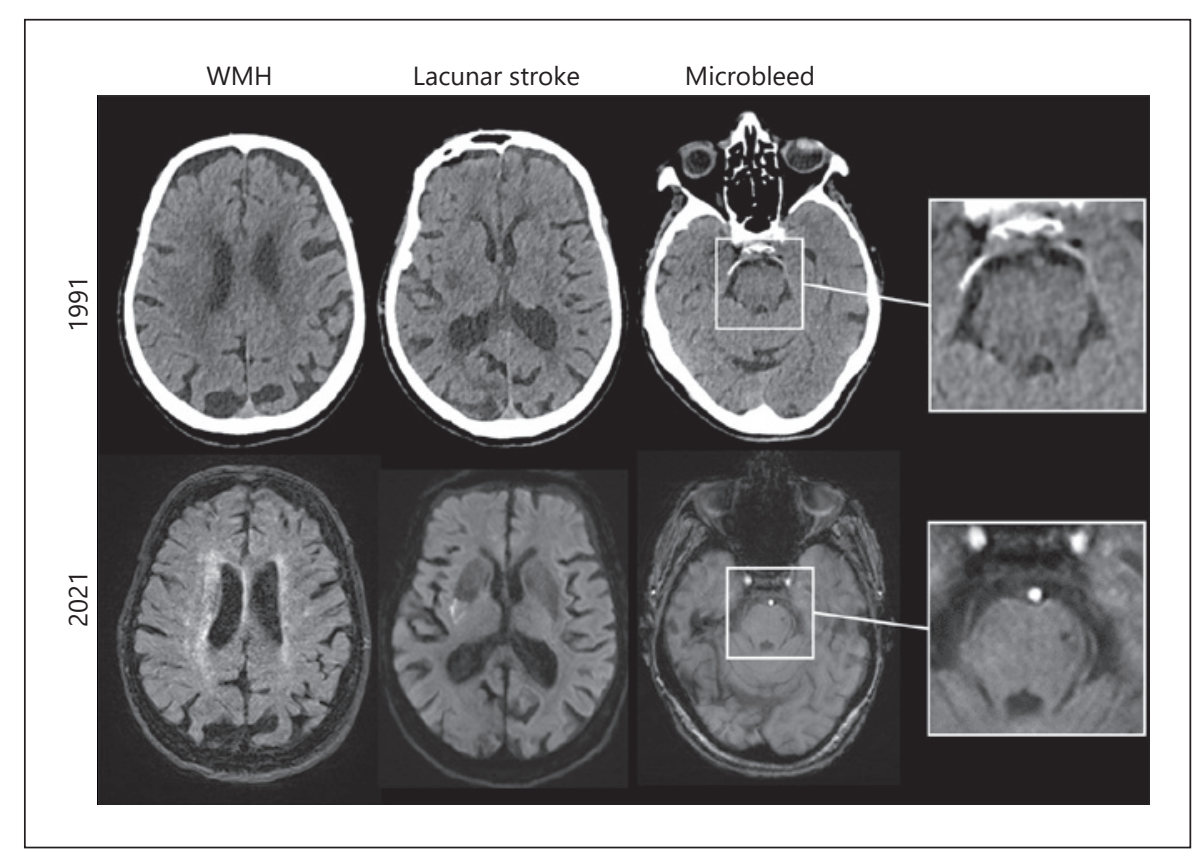

subcortical infarcts and leukoencephalopathy (CADASIL), the most frequent monogenic form of cSVD, which has made it possible to build large cohorts of patients with the same pure disorder to comprehensively describe its clinical and radiological spectrum and better understand the natural history and pathophysiology of the most common forms of cSVD.

\section{The Advent of Brain MRI in the Early 1990s Significantly Changed Daily Clinical Practice}

\section{An Increased Interest in Chronic White Matter Changes}

The first MRI scanner was installed in the clinical setting in 1984, but it remained rarely available before the 1990s. With the generalization of brain MRI, the interest for white matter changes increased drastically. The prevalence of such changes on the CT scan, i.e., leukoaraiosis, has been estimated to be approximately $50 \%$ in the elderly [3]. With MRI, this estimate has increased dramatically to $80-90 \%$ after the age of 65 years, with enormous variability in their extent between individuals, making white matter changes one of the most intriguing MRI markers of interactions between aging and vascular risk factors, particularly hypertension [4] (Fig. 1). This has shed new light on that domain, attracting the interest of vascular neurologists, epidemiologists, geriatricians, etc. However, it was not until long after, in 2013, after count- less terms from neuropathology or radiology had been used interchangeably for decades to describe the same thing (vascular leukoencephalopathy, leukoaraiosis, Binswanger encephalopathy, subcortical arteriosclerotic encephalopathy, etc.) that STRIVE led to widespread adoption of the term white matter hyperintensities (WMHs) to describe these MRI changes. This term had the major advantage to be purely descriptive and not to try to address the nature of underlying tissue alterations which are now known to be highly variable from one brain area to another and from patient to patient [2].

\section{A New Understanding of Acute Ischemic Events Related to cSVD}

In parallel with the generalization of MRI and the growing interest for $\mathrm{WMH}$ and their clinical relevance, the advent of diffusion-weighted imaging (DWI), introduced into the clinical setting in the late 1990s [5], has revolutionized the management of cSVD-related ischemic stroke. Historically, before the advent of the CT scan, patients with acute stroke were sometimes treated with heparin on the basis of clinical assessment alone, for example, in the case of a Wallenberg syndrome when intracerebral hemorrhage (ICH) was considered highly unlikely [6]. This attitude would of course be unthinkable nowadays. The advent of the CT scan allowed in the 1970s to systematically exclude ICH before treating patients [7], but the revolution happened in the late 1990s, when small-sized ischemic alterations became visible in the 
acute phase [8]. Until then, the knowledge of lacunar syndromes was considered crucial for the accurate diagnosis of ischemic stroke related to cSVD as they were likely to be related to the occlusion of a single perforating artery and in contrast unlikely related to ICH $[9,10]$. In the 1990 s, it was very common in medical training to ask students to learn the specifics of countless lacunar syndromes. Some 20 years later, the interest for lacunar syndromes when a DWI lesion is present on MRI has decreased. Lacunar syndromes may still be of importance to help diagnosing acute ischemic stroke in the absence of a DWI lesion, given that MRI is not fully sensitive to small ischemic changes, particularly in the first $24 \mathrm{~h}$ in the posterior circulation [11]. Besides, elderly patients may present with counter-indication to brain MRI. Interestingly, in our experience, the ease with which the diagnosis of ischemic stroke is retained on the sole presence of a lacunar syndrome in the absence of a DWI lesion is highly dependent on the local habits. To note, recent data have shown these lacunar syndrome are not specific to cSVDrelated ischemic stroke but can also be seen in cortical ischemic stroke as well as in the acute phase of ICH [12]. Beyond that, clinical symptoms associated with recent small subcortical infarcts, which currently define the lacunar syndrome, will probably have to be renamed because lacunar refers to the Latin root lacuna, meaning hole, which is no longer relevant. However, we are not aware of a widely accepted alternative term.

\section{A Novel Interest in cSVD-Related Acute Ischemic}

Lesions without Stroke Symptoms

Punctate bright spots on DWI with a reduced apparent diffusion coefficient, in all respects like those observed in ischemic stroke, were finally also observed in the absence of stroke symptoms in cSVD. Some authors have even suggested that, given their high frequency, these spots may contribute to the development of $\mathrm{WMH}$, but these results have not been replicated to date, and an overrepresentation of patients with cerebral amyloid angiopathy in these studies may well have led to a biased interpretation [13]. Systematic MRI studies also revealed that such lesions, not associated with neurological deficits, were often visible remote to the site of acute ICH [14]. While this finding was initially thought to be related to the underlying cause of $\mathrm{ICH}$, and even sometime suggestive of infectious endocarditis, it was finally shown that the presence of such alterations was in fact related to the actual severity, cause, and pathophysiology of the underlying cSVD [15]. A number of questions remain as to why some of these lesions are associated with stroke symptoms while others are not. While it may seem obvious that this difference is related to the fact that some lie in neurologically eloquent areas (motor, visual, and sensory) and others do not, a study conducted in CADASIL found that the location was not predictive of the presence of stroke symptoms [16]. Altogether, DWI in cSVD allowed to show that acute ischemic manifestations in CSVD are not always associated with stroke symptoms but most often highly suggestive of a diffuse brain involvement. It is noteworthy that in CADASIL, clinical severity is only weakly related to stroke, suggesting that the total tissue damage burden may be more important than the impact of acute stroke on disease severity in cSVD [17].

\section{Cerebral Microbleeds, a Novel MRI Marker of}

Hemorrhagic and Ischemic Strokes

Another major evolution with brain MRI was the identification of cerebral microbleeds (CMs) on sequences sensitive to susceptibility artefacts [18-20]. Completely invisible on the CT scan, CMs were rapidly found important for patient care. The number of CMs was rapidly found related to the risk of first-ever or recurrent ICH [21], while their distribution (cortical, deep, or both) was found linked to the underlying CSVD [22]. More recently however, studies have shown that CMs are also an independent risk marker of ischemic events [23], a finding that can be explained by the fact that underlying tissue lesions are not always hemorrhagic, but a significant proportion of them may also be ischemic in nature [24]. Recently, the Microbleeds International Collaborative Network initiative allowed to build and validate a score to predict the risk of ischemic and hemorrhagic stroke in patients with previous ischemic stroke or transient ischemic attack. In line with the abovementioned, somehow counter-intuitive results, the risk of ischemic stroke in these patients is higher in those with larger numbers of CMs [25].

\section{The Building of Large Cohorts of CADASIL in the Late 1990s Allowed Defining Homogeneous Populations to Better Describe the Spectrum of cSVD}

\section{Identification of the NOTCH3 Gene as the Cause of CADASIL}

The race after the identification of the first families with hereditary cSVD began in the 1970s, in particular with the seminal work of Marie-Germaine Bousser and coworkers [26]. In the 1990s, the spectrum of cSVD was mostly inferred from Fisher's [27] seminal neuropatho- 


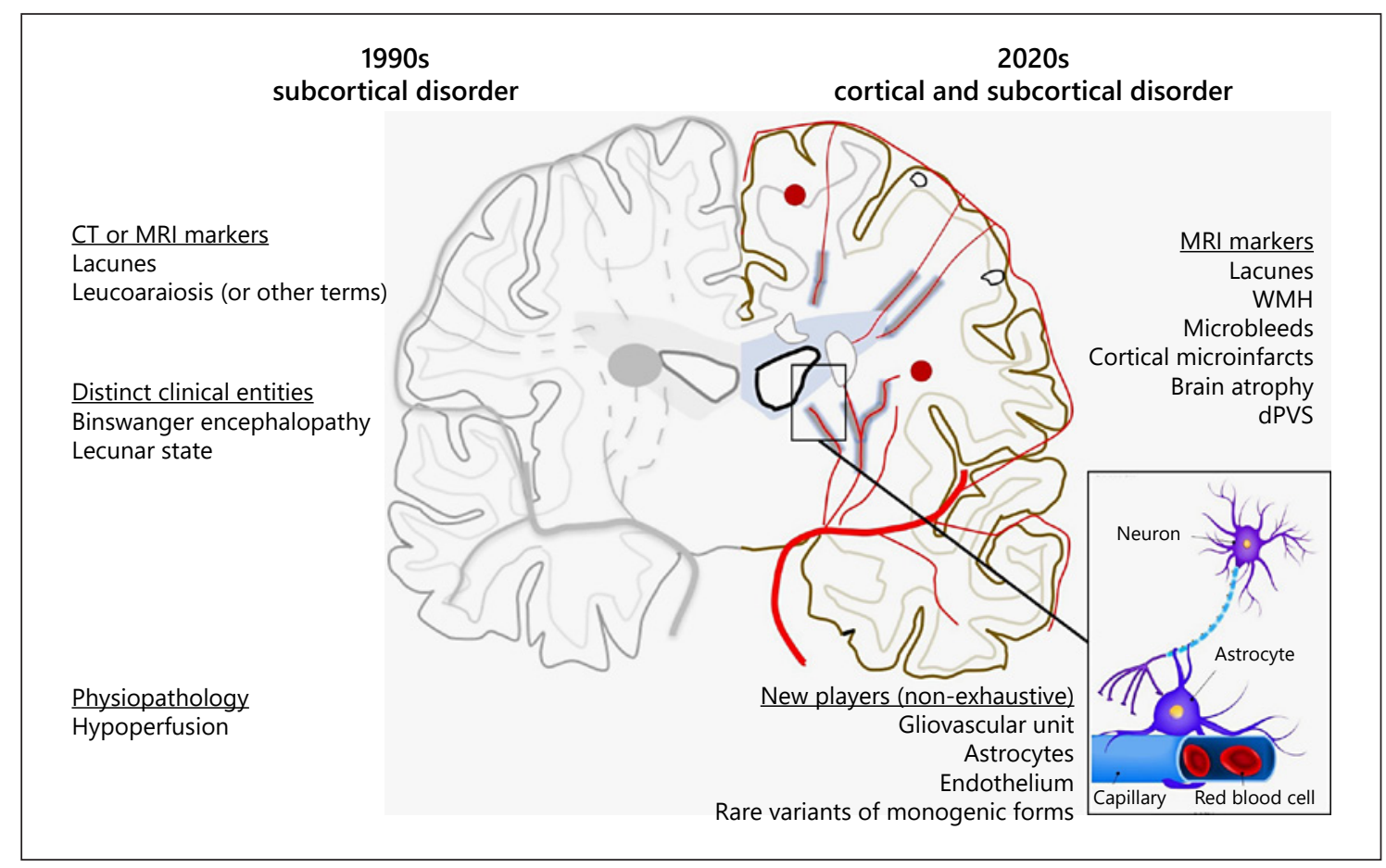

Fig. 2. Schematic representation of key points in the understanding of cSVD mechanisms over the past 30 years. MRI, magnetic resonance imaging; cSVD, cerebral small-vessel diseases; WMH, white mater hyperintensity; dPVS, dilatated perivascular spaces.

logical work on sporadic forms of cSVD and the radiological descriptions of various degrees of leukoaraïosis and numbers of lacunes on the CT scan [28]. Then, the gene of CADASIL, the most frequent monogenic cSVD, was identified [29], which allowed gathering data from patients affected by the disorder, leading in the 2000s to the Paris-Munich cohort in which were collected the data of several hundreds of prospectively recruited patients [30].

\section{A Better Description of the Clinico-Radiological Spectrum of cSVD in General}

A major consequence of such large cohorts was the possibility to describe the natural clinical and radiological history of a pure model of cSVD. While initial reports were naturally biased towards more severe forms (easier to diagnose), with $60 \%$ of patients being described as dead or disabled at the age of 60 years [31], the progressive inclusion of first-degree relatives, in whom the diagnosis was made in various contexts, including the total absence of overt symptom, allowed tending towards the full spectrum of disease severity.

With the use of systematic and standardized brain MRI, a first critical impact was the demonstration of the wideness of the radiological spectrum of CADASIL, even within families in individuals sharing the same mutation. Progressively, the concept of lacunar state [32] and Binswanger encephalopathy [33], long considered to be 2 distinct entities [34, 35], appeared as 2 extremes from the spectrum of cSVD. It is however still not understood why the radiological spectrum of cSVD may vary in such extents within families sharing the same mutation. Interestingly, monogenic forms related to mutations upregulating COLIVA1 leading to the PADMAL phenotype with multiple pontine lacunes may help in the future understand why some patients have more lacunes or more WMH [36].

\section{A Better Understanding of the Cognitive and}

Behavioral Symptoms Actually Related to cSVD

Another major impact of these cohorts was the description of the cognitive and behavioral consequences of cSVD independent of concomitant neurodegenerative diseases, particularly Alzheimer's disease (AD), at a time when neither cerebrospinal fluid biomarkers nor PIB PET were available to rule out concomitant $\mathrm{AD}$. As in younger patients, the likelihood of being also affected by concomitant $\mathrm{AD}$ is very low, and the neurocognitive profile of pure cSVD 
and its relationships with brain MRI could be described [37] and thoroughly compared with sporadic forms [38]. Later, the comparison of mildly affected patients to age- and sex-matched healthy subjects allowed showing that processing speed slowing in the order of milliseconds may be the first manifestation in pure cSVD [39].

\section{From Subcortical to Global Brain Disorder}

More generally, until late 2000s, the fact that most visible lesions, both on MRI and on neuropathology, lie in subcortical areas made of cSVD a typical subcortical disorder, but the situation has now profoundly changed (Fig. 2). The identification of cortical microinfarcts, which have rapidly become a major concern because of their frequency and their supposedly large impact on local cortical functioning [40], has played an important role in the recognition of cortical involvement in CSVD. The direct involvement of the cerebral cortex in CSVD was confirmed by the demonstration of early and diffusive alterations in the cortical mantle during the course of CADASIL, as well as sporadic CSVD, in parallel with the cognitive deterioration $[41,42]$. A clear link between subcortical macroscopic lesions and cortical alterations was then demonstrated elegantly with the use of advanced imaging approaches [43].

\section{New Insights into the Pathophysiology of CSVD}

Until the 2000s, the pathophysiology of cSVD was thought mostly related to hypoperfusion. Indeed, in the most common causes of cSVD, WMH initially appear in watershed areas in the deep brain regions, where the density of arterioles is the lowest, with rare collaterals [44]. In line, ischemic stroke would result from the occlusion of a single perforating artery. The identification of monogenic forms of cSVD, the development of animal models of these monogenic forms, in particular CADASIL, and advances in various fields in relation to cSVD were identified more or less deliberately and have fundamentally altered the understanding of cSVD pathophysiology. Aside from chronic hypoperfusion, a number of mechanisms were progressively shown involved in CSVD, including a key role of the endothelium, astrocytes, the gliovascular system, perivascular spaces, etc. [2]. This paradigmatic shift can be easily illustrated by the case report of a CADASIL patient who had a strong and reversible reduction of the volume of her WMH while undertaking for months valpromide which is known to interact with aquaporin 4 protein on astrocytic endfeet, suggesting that variations in water content between different compartments in the brain might underlie some WMH in cSVD [45]. In line, as chronic hypoperfusion could probably not explain per se the formation of lesions underlying WMH, the occlusion of a single perforating artery could probably not explain the occurrence of cSVD-related ischemic strokes. Even with the most comprehensive neuropathological evaluation, the presence of thrombus occluding perforating arteries has not been demonstrated [46]. This has led researchers to find alternative hypotheses. One of them is related to endothelial dysfunction, and some authors have reported diffuse alterations of the blood-brain barrier in the acute phase of ischemic stroke related to cSVD [47]. To date, however, the mechanisms leading to the acute ischemic stroke in cSVD remain largely undetermined.

Beyond these new leads originating from animal models and neuropathology, the role, in the general population, of rare variants of the genes responsible for monogenic forms of cSVD have been highlighted thanks to the exploitation of large genetic databases, in particular with the use of genome-wide association studies. The presence of NOTCH3 rare variants was for instance shown associated with larger burden of WMH [48]. Similar results were observed recently for rare variants in the HTRA1 gene, which has a particular positioning in the field of monogenic cSVD. Indeed, while bi-allelic mutations of HTRA 1 are responsible for the exceptional cerebral autosomal recessive arteriopathy with subcortical infarcts and leukoencephalopathy syndrome, autosomal dominant mutations have also been reported and may be the second most frequent form of hereditary cSVD [49]. Noteworthily, the presence of a rare variant in HTRA1 may have a larger effect size on the WMH burden than the diagnosis of hypertension [50].

\section{Conclusion}

In this opinion paper, we aimed to illustrate how MRI, particularly diffusion, and the building of large cohorts of patients with CADASIL as a model to study the more common forms of cSVD have profoundly changed the perception of their pathophysiology. Less than 30 years ago, their presumed pathophysiology was overly simplistic around hypoperfusion, some of the different facets of cSVD were sometimes considered independent disorders, and understanding of the disease was largely affected by assessment biases due to technical limitations. However, despite enormous progress, major gaps remain in the understanding of cSVD, and key questions, such as the nature of the mechanisms leading to ischemic stroke 
in CSVD, remain unanswered. Hopefully, these answers will be put in perspective for the 50th anniversary of Cerebrovascular Diseases.

\section{Conflict of Interest Statement}

The authors have no conflicts of interest to declare.

\section{Funding Sources}

This work was supported by a grant from the "Agence Régional de Santé Ile de France" to Lina Grosset and a grant from the "Fondation pour la Recherche sur les AVC" to Eric Jouvent.

\section{Author Contributions}

Lina Grosset drafted the manuscript. Eric Jouvent corrected the manuscript.

\section{References}

1 Wardlaw JM, Smith EE, Biessels GJ, Cordonnier C, Fazekas F, Frayne R, et al. Neuroimaging standards for research into small vessel disease and its contribution to ageing and neurodegeneration. Lancet Neurol. 2013; 12(8):822-38.

2 Wardlaw JM, Smith C, Dichgans M. Small vessel disease: mechanisms and clinical implications. Lancet Neurol. 2019;18(7):684-96.

3 Boone KB, Miller BL, Lesser IM, Mehringer CM, Hill-Gutierrez E, Goldberg MA, et al. Neuropsychological correlates of white-matter lesions in healthy elderly subjects. A threshold effect. Arch Neurol. 1992;49(5): 549-54.

4 Richard E, Gouw AA, Scheltens P, van Gool WA. Vascular care in patients with Alzheimer disease with cerebrovascular lesions slows progression of white matter lesions on MRI: the evaluation of vascular care in Alzheimer's disease (EVA) study. Stroke. 2010;41:554-6.

5 Albers GW. Diffusion-weighted MRI for evaluation of acute stroke. Neurology. 1998; 51(3 Suppl 3):S47-9.

6 Magnusson JH. Thrombosis of the posteriorinferior cerebellar artery (Wallenberg syndrome) treated by heparin. Lancet. 1938; 1(5977):664-5.

7 Mori E, Tabuchi M, Yamadori A. Lacunar syndrome due to intracerebral hemorrhage. Stroke. 1985;16(3):454-9.

8 van Everdingen KJ, van der Grond J, Kappelle LJ, Ramos LM, Mali WP. Diffusion-weighted magnetic resonance imaging in acute stroke. Stroke. 1998;29(9):1783-90.

9 Fisher CM. Lacunes: small, deep cerebral infarcts. Neurology. 1965;15(8):774-84.

10 Fisher CM. Pure sensory stroke and allied conditions. Stroke. 1982;13(4):434-47.

11 Edlow BL, Hurwitz S, Edlow JA. Diagnosis of DWI-negative acute ischemic stroke: a metaanalysis. Neurology. 2017;89(3):256-62.

12 Arboix A, Massons J, García-Eroles L, Targa C, Comes E, Parra O. Clinical predictors of lacunar syndrome not due to lacunar infarction. BMC Neurol. 2010;10:31

13 Conklin J, Silver FL, Mikulis DJ, Mandell DM. Are acute infarcts the cause of leukoaraiosis? Brain mapping for 16 consecutive weeks. Ann Neurol. 2014;76(6):899-904.
14 Gregoire SM, Charidimou A, Gadapa N, Dolan E, Antoun N, Peeters A, et al. Acute ischaemic brain lesions in intracerebral haemorrhage: multicentre cross-sectional magnetic resonance imaging study. Brain. 2011; 134(Pt 8):2376-86.

15 Wiegertjes K, Dinsmore L, Drever J, Hutchison A, Stephen J, Valdés Hernández MC, et al. Diffusion-weighted imaging lesions and risk of recurrent stroke after intracerebral haemorrhage. J Neurol Neurosurg Psychiatry. 2021;92(9):950-5.

16 Osman O, De Guio F, Chabriat H, Jouvent E. Why are only some subcortical ischemic lesions on diffusion magnetic resonance imaging associated with stroke symptoms in small vessel disease? Stroke. 2018;49(8):1920-3.

17 Chabriat H, Hervé D, Duering M, Godin O, Jouvent E, Opherk C, et al. Predictors of clinical worsening in cerebral autosomal dominant arteriopathy with subcortical infarcts and leukoencephalopathy: Prospective Cohort Study. Stroke. 2016;47(1):4-11.

18 Roob G, Fazekas F. Magnetic resonance imaging of cerebral microbleeds. Curr Opin Neurol. 2000;13(1):69-73.

19 Lesnik Oberstein SA, van den Boom R, van Buchem MA, van Houwelingen HC, Bakker $\mathrm{E}$, Vollebregt E, et al. Cerebral microbleeds in CADASIL. Neurology. 2001;57(6):1066-70.

20 Dichgans M, Holtmannspötter M, Herzog J, Peters N, Bergmann M, Yousry TA. Cerebral microbleeds in CADASIL: a gradient-echo magnetic resonance imaging and autopsy study. Stroke. 2002;33(1):67-71.

21 Fan YH, Zhang L, Lam WW, Mok VC, Wong KS. Cerebral microbleeds as a risk factor for subsequent intracerebral hemorrhages among patients with acute ischemic stroke. Stroke. 2003;34(10):2459-62.

22 Pasi M, Charidimou A, Boulouis G, Auriel E, Ayres A, Schwab KM, et al. Mixed-location cerebral hemorrhage/microbleeds: underlying microangiopathy and recurrence risk. Neurology. 2018;90(2):e119-26.
23 Wilson D, Ambler G, Lee KJ, Lim JS, Shiozawa M, Koga M, et al. Cerebral microbleeds and stroke risk after ischaemic stroke or transient ischaemic attack: a pooled analysis of individual patient data from cohort studies. Lancet Neurol. 2019;18(7):653-65.

24 Chang R, Castillo J, Zambon AC, Krasieva TB, Fisher MJ, Sumbria RK. Brain endothelial erythrophagocytosis and hemoglobin transmigration across brain endothelium: implications for pathogenesis of cerebral microbleeds. Front Cell Neurosci. 2018;12:279.

25 Best JG, Ambler G, Wilson D, Lee KJ, Lim JS, Shiozawa M, et al. Development of imagingbased risk scores for prediction of intracranial haemorrhage and ischaemic stroke in patients taking antithrombotic therapy after ischaemic stroke or transient ischaemic attack: a pooled analysis of individual patient data from cohort studies. Lancet Neurol. 2021; 20(4):294-303.

26 Tournier-Lasserve E, Iba-Zizen MT, Romero N, Bousser MG. Autosomal dominant syndrome with strokelike episodes and leukoencephalopathy. Stroke. 1991;22(10):1297-302.

27 Fisher CM. The arterial lesions underlying lacunes. Acta Neuropathol. 1968;12(1):1-15.

28 Miyao S, Takano A, Teramoto J, Takahashi A. Leukoaraiosis in relation to prognosis for patients with lacunar infarction. Stroke. 1992; 23(10): 1434-8.

29 Joutel A, Corpechot C, Ducros A, Vahedi K, Chabriat $\mathrm{H}$, Mouton P, et al. Notch3 mutations in CADASIL, a hereditary adult-onset condition causing stroke and dementia. $\mathrm{Na}-$ ture. 1996;383(6602):707-10.

30 Duering M, Csanadi E, Gesierich B, Jouvent E, Hervé D, Seiler S, et al. Incident lacunes preferentially localize to the edge of white matter hyperintensities: insights into the pathophysiology of cerebral small vessel disease. Brain. 2013;136(Pt 9):2717-26.

31 Di Donato I, Bianchi S, De Stefano N, Dichgans M, Dotti MT, Duering M, et al. Cerebral autosomal dominant arteriopathy with subcortical infarcts and leukoencephalopathy (CADASIL) as a model of small vessel disease: update on clinical, diagnostic, and management aspects. BMC Med. 2017;15(1):41. 
32 Katzman R. Dementias. Postgrad Med. 1978; 64(2):119-25.

33 Babikian V, Ropper AH. Binswanger's disease: a review. Stroke. 1987;18(1):2-12.

34 Litvak LB, Niagu-Beliaeva AI. The "lacunar state" of the brain in the atherosclerotic phase of hypertension. Zh Nevropatol Psikhiatr Im S S Korsakova. 1970;70(6):801-8.

35 Caplan LR, Schoene WC. Clinical features of subcortical arteriosclerotic encephalopathy (Binswanger disease). Neurology. 1978; 28(12):1206-15.

36 Verdura E, Hervé D, Bergametti F, Jacquet C, Morvan T, Prieto-Morin C, et al. Disruption of a miR-29 binding site leading to COL4A1 upregulation causes pontine autosomal dominant microangiopathy with leukoencephalopathy. Ann Neurol. 2016;80(5):741-53.

37 Buffon F, Porcher R, Hernandez K, Kurtz A, Pointeau S, Vahedi K, et al. Cognitive profile in CADASIL. J Neurol Neurosurg Psychiatry. 2006;77(2):175-80.

38 Charlton RA, Morris RG, Nitkunan A Markus HS. The cognitive profiles of CADASIL and sporadic small vessel disease. Neurology. 2006;66(10):1523-6.

39 Jouvent E, Reyes S, De Guio F, Chabriat H. Reaction time is a marker of early cognitive and behavioral alterations in pure cerebral small vessel disease. J Alzheimers Dis. 2015; 47(2):413-9.

40 van Veluw SJ, Shih AY, Smith EE, Chen C, Schneider JA, Wardlaw JM, et al. Detection, risk factors, and functional consequences of cerebral microinfarcts. Lancet Neurol. 2017; 16(9):730-40.

41 Peres R, De Guio F, Chabriat H, Jouvent E. Alterations of the cerebral cortex in sporadic small vessel disease: a systematic review of in vivo MRI data. J Cereb Blood Flow Metab. 2016;36(4):681-95.

42 Lambert C, Benjamin P, Zeestraten E, Lawrence AJ, Barrick TR, Markus HS. Longitudinal patterns of leukoaraiosis and brain atrophy in symptomatic small vessel disease. Brain. 2016;139(Pt 4):1136-51.

43 Duering M, Righart R, Csanadi E, Jouvent E, Hervé $\mathrm{D}$, Chabriat $\mathrm{H}$, et al. Incident subcortical infarcts induce focal thinning in connected cortical regions. Neurology. 2012;79(20):2025-8.

44 Duering M, Csanadi E, Gesierich B, Jouvent E, Hervé D, Seiler S, et al. Incident lacunes preferentially localize to the edge of white matter hyperintensities: insights into the pathophysiology of cerebral small vessel disease. Brain. 2013;136(9):2717-26.

45 Jouvent E, Alili N, Hervé D, Chabriat H. Vanishing white matter hyperintensities in cada- sil: a case report with insight into disease mechanisms. J Alzheimers Dis. 2020;78(3): 907-10.

46 Wardlaw JM, Dennis MS, Warlow CP, Sandercock PA. Imaging appearance of the symptomatic perforating artery in patients with lacunar infarction: occlusion or other vascular pathology? Ann Neurol. 2001;50(2):208-15.

47 Wardlaw JM, Sandercock PA, Dennis MS, Starr J. Is breakdown of the blood-brain barrier responsible for lacunar stroke, leukoaraiosis, and dementia? Stroke. 2003;34(3):80612.

48 Mishra A, Chauhan G, Violleau MH, Vojinovic D, Jian X, Bis JC, et al. Association of variants in HTRA1 and NOTCH3 with MRIdefined extremes of cerebral small vessel disease in older subjects. Brain. 2019;142(4): 1009-23.

49 Verdura E, Hervé D, Scharrer E, Amador Mdel M, Guyant-Maréchal L, Philippi A, et al. Heterozygous HTRA1 mutations are associated with autosomal dominant cerebral small vessel disease. Brain. 2015;138(Pt 8):2347-58.

50 Malik R, Beaufort N, Frerich S, Gesierich B, Georgakis MK, Rannikmäe K, et al. Wholeexome sequencing reveals a role of HTRA1 and EGFL 8 in brain white matter hyperintensities. Brain. 2021;144(9):2670-82. 